\title{
Differentiable comparative statics with payoff functions not differentiable everywhere
}

\author{
Luis C. Corchon* \\ Universidad Carlos III de Madrid, Departamento de Economia, Calle Madrid, 126, Getafe 28903, Madrid, Spain
}

Received 4 December 2000; accepted 30 January 2001

\begin{abstract}
In this note we consider Cournot oligopoly. Due to the consideration of several consumers and/or technologies, the profit function is not differentiable everywhere. We show that Cournot equilibrium never occurs in outputs where profit functions are not-differentiable. This result validates the procedure of differentiating profit functions to perform comparative statics in models where profit functions are not differentiable everywhere. (C) 2001 Elsevier Science B.V. All rights reserved.
\end{abstract}

Keywords: Comparative statics; Differentiability

JEL classification: L13

\section{The model and the results}

Consider a market where the demand function is linear, i.e. $x=a-b p$, where $x$ is aggregate output and $p$ is the market price. However, the assumption of linearity cannot hold everywhere because for $p>a / b$ demand is negative. In order to avoid negative demand we may write the demand function as $x=\max \{0, a-b p\}$. Thus, the demand function is not differentiable at $p=0$. However, as long as equilibrium occurs at an allocation yielding a positive price, the demand function is differentiable.

Suppose now that the above demand function corresponds to a given country, say $\mathrm{C}$, and this country enters into a free trade zone with demand function (before the entry of $\mathrm{C}$ ) equal to $\max \{0, A-B p\}$ with $A>a$ and $A / B>a / b$. Thus, the demand function for the free trade zone, once country $\mathrm{C}$ has entered is

$$
x=0 \text { for } p>A / B \text {. }
$$

*Tel.: +34-91-624-9617; fax: +34-91-624-9875.

E-mail address: lcorchon@eco.uc3m.es (L.C. Corchon). 


$$
\begin{aligned}
& x=A-B p \quad \text { for } a / b \leq p \leq A / B \\
& x=a+A-(b+B) p \quad \text { for } p<a / b .
\end{aligned}
$$

The new demand function is not differentiable at $p=a / b$. Thus, if equilibrium occurs at an allocation yielding this price, payoff functions are not differentiable there. Since most results in comparative statics are obtained differentiating profit functions, these results cannot be applied to the model above. Fortunately, we will see that if demand (or cost) is continuously differentiable almost everywhere, Cournot equilibrium always occurs at differentiable points.

Let $p=p(x)$ be the inverse demand function. Let $C_{i}\left(x_{i}\right)$ be the cost function of firm $i$, where $x_{i}$ is the output of firm $i$.

Definition. A Cournot Equilibrium is a list of outputs $\left(\hat{x}_{1}, \ldots, \hat{x}_{n}\right)$ such that $\forall i=1, \ldots, n$,

$$
p\left(\sum_{j=1}^{n} \hat{x}_{j}\right) \hat{x}_{i}-C_{i}\left(\hat{x}_{i}\right) \geq p\left(x_{i}+\sum_{j \neq i} \hat{x}_{j}\right) x_{i}-C_{i}\left(x_{i}\right), \forall x_{i} \in \mathbb{R}_{+} .
$$

Suppose $p()$ is not differentiable at the Cournot equilibrium aggregate output $\sum_{j=1}^{n} \hat{x}_{j} \equiv \hat{x}$, but there exists a $\bar{\epsilon}>0$ such that $p()$ is continuously differentiable in $(\hat{x}, \hat{x}+\epsilon)$ and $(\hat{x}, \hat{x}-\epsilon)$ for all $\epsilon \in(0, \bar{\epsilon})$. If this lack of differentiability is caused by the aggregation of different demand curves it must be that

$$
\frac{\partial p(\hat{x}+\delta)}{\partial x}>\frac{\partial p(\hat{x}-\delta)}{\partial x}
$$

for all $\delta$ sufficiently small but positive.

The above assumption just generalizes the fact that the piecewise linear demand function above was continuously differentiable at all interior points except at $p=a / b$ and that computing the slope of the inverse demand function in the linear model above,

$$
\frac{\partial p(\hat{x}+\delta)}{\partial x}=-\frac{1}{b+B}>-\frac{1}{B}=\frac{\partial p(\hat{x}-\delta)}{\partial x} .
$$

Let

$$
\frac{\partial p\left(\hat{x}-\mathrm{d} x_{i}\right)}{\partial x} \equiv \lim _{\delta \rightarrow 0} \frac{\partial p(\hat{x}-\delta)}{\partial x} .
$$

Consider now that firm $i$ decreases infinitesimally its equilibrium output from the one given by the C.E. by $\mathrm{d} x_{i}$. Since $\hat{x}$ is a C.E., this deviation cannot be profitable, i.e.

$$
\mathrm{d} U_{i}=\mathrm{d} x_{i}\left(\frac{\partial p\left(\hat{x}-\mathrm{d} x_{i}\right)}{\partial x} \hat{x}_{i}+p(\hat{x})-\frac{\partial C_{i}\left(\hat{x}_{i}\right)}{\partial x_{i}}\right) \leq 0,
$$

or

$$
\frac{\partial p\left(\hat{x}-\mathrm{d} x_{i}\right)}{\partial x} \hat{x}_{i}+p(\hat{x})-\frac{\partial C_{i}\left(\hat{x}_{i}\right)}{\partial x_{i}} \geq 0
$$

Similarly, let 


$$
\frac{\partial p\left(\hat{x}+\mathrm{d} x_{i}\right)}{\partial x} \equiv \lim _{\delta \rightarrow 0} \frac{\partial p(\hat{x}+\delta)}{\partial x}
$$

Again, if firm $i$ increases infinitesimally its output from the one given by the C.E. by $\mathrm{d} x_{i}$, profits cannot increase, i.e.

$$
\mathrm{d} U_{i}=\mathrm{d} x_{i}\left(\frac{\partial p\left(\hat{x}+\mathrm{d} x_{i}\right)}{\partial x} \hat{x}_{i}+p(\hat{x})-\frac{\partial C_{i}\left(\hat{x}_{i}\right)}{\partial x_{i}}\right) \leq 0
$$

or

$$
\frac{\partial p\left(\hat{x}+\mathrm{d} x_{i}\right)}{\partial x} \hat{x}_{i}+p(\hat{x})-\frac{\partial C_{i}\left(\hat{x}_{i}\right)}{\partial x_{i}} \leq 0
$$

Thus

$$
\frac{\partial p\left(\hat{x}-\mathrm{d} x_{i}\right)}{\partial x} \geq \frac{\partial p\left(\hat{x}+\mathrm{d} x_{i}\right)}{\partial x}
$$

contradicting our initial assumption. Thus, at least one of the deviations must be profitable and the C.E. cannot occur at this allocation.

A similar analysis can be made in the case in which the cost function is not differentiable everywhere. Suppose again that there is a C.E. with aggregate output $\hat{x}$ and individual outputs $\left(\hat{x}_{1}, \ldots, \hat{x}_{n}\right)$. At output $\hat{x}_{i}$, the cost function is not differentiable. In particular for outputs smaller than $\hat{x}_{i}$, the cheaper production process is one suitable for small scale of operations with marginal cost denoted by $\partial C_{i}() / \partial x_{i}$. For outputs larger than $\hat{x}_{i}$, the cheaper production process is one suitable for mass production with marginal cost denoted by $\partial K_{i}() / \partial x_{i}{ }^{1}$. We assume that

$$
\frac{\partial K_{i}\left(\hat{x}_{i}\right)}{\partial x_{i}}<\frac{\partial C_{i}\left(\hat{x}_{i}\right)}{\partial x_{i}}
$$

i.e. the mass production process has smaller marginal costs at $\hat{x}_{i}$. Suppose now that firm $i$ decreases infinitesimally its equilibrium output by $\mathrm{d} x_{i}$. Since we start at a C.E., this deviation cannot be profitable,

$$
\mathrm{d} U_{i}=\mathrm{d} x_{i}\left(\frac{\partial p(\hat{x})}{\partial x} \hat{x}_{i}+p(\hat{x})-\frac{\partial C_{i}\left(\hat{x}_{i}\right)}{\partial x_{i}}\right) \leq 0,
$$

or

$$
\frac{\partial p(\hat{x})}{\partial x} \hat{x}_{i}+p(\hat{x})-\frac{\partial C_{i}\left(\hat{x}_{i}\right)}{\partial x_{i}} \geq 0 .
$$

Similarly, if firm $i$ increases infinitesimally its output by $\mathrm{d} x_{i}$,

\footnotetext{
${ }^{1}$ Imagine that the mass-production technique has a higher fixed cost than the other production process. Thus, only if the output is large enough does it pay to use the mass-production technique.
} 


$$
\mathrm{d} U_{i}=\mathrm{d} x_{i}\left(\frac{\partial p(\hat{x})}{\partial x} \hat{x}_{i}+p(\hat{x})-\frac{\partial K_{i}\left(\hat{x}_{i}\right)}{\partial x_{i}}\right) \leq 0
$$

or

$$
\frac{\partial p(\hat{x})}{\partial x} \hat{x}_{i}+p(\hat{x})-\frac{\partial K_{i}\left(\hat{x}_{i}\right)}{\partial x_{i}} \leq 0 .
$$

Thus,

$$
\frac{\partial K_{i}\left(\hat{x}_{i}\right)}{\partial x_{i}} \geq \frac{\partial C_{i}\left(\hat{x}_{i}\right)}{\partial x_{i}}
$$

contradicting our initial assumption. Thus, at least one of the deviations must be profitable and the C.E. cannot occur at this allocation

\section{Discussion of the results}

In this note we have shown that in a certain class of models, lack of differentiability everywhere is not a problem in order to perform comparative statics using differentiability because equilibrium never occurs at non-differentiable points. The intuition of this result is simple: Consider a monopoly with zero production costs. It is very clear that maximum revenue cannot occur at the point in which the demand function has the kind of kink we are assuming.

It might be argued that it is very unlikely that equilibrium occurs at points which are of zero measure. However this argument is not correct: For instance, consider a one household economy with a non differentiable production function. It is easy to see in a picture that competitive equilibrium may occur at non-differentiable points for a non negligible set of preferences. The same thing occurs in linear programming where extrema occur very often at non differentiable points. Thus, points which, a priori, look very unlikely to be good candidates for equilibrium allocations are in fact good candidates for such allocations. In this note we have shown that, for one class of models, this is not the case. Notice that the condition

$$
\frac{\partial p(\hat{x}+\delta)}{\partial x}>\frac{\partial p(\hat{x}-\delta)}{\partial x},
$$

is essential for our results to hold. If this inequality is reversed, it is easy to show that there are C.E. at points where the profit function is not differentiable. Moreover our result is not an "almost everywhere" result. It says that C.E. never occurs at non differentiable points.

Notice the condition above implies that the demand curve is convex. Thus, profit functions are not concave and this raises a doubt on the possible lack of existence of C.E.. However, existence of a C.E. can be established, even if profit functions are not concave: For instance if all firms are identical (Mc Manus, 1962, 1964) or if $n=2$ (Vives, 1990). 


\section{References}

Mc Manus, M., 1962. Numbers and size in Cournot oligopoly. Yorkshire Bull 14, 14-22.

Mc Manus, M., 1964. Equilibrium, numbers and size in Cournot oligopoly. Yorkshire Bull 16, 68-75.

Vives, X., 1990. Nash equilibrium with strategic complementarities. Journal of Mathematical Economics 19, 305-321. 